\title{
Sex Differences in Reported Adverse Drug Reactions to COVID-19 Drugs in a Global Database of Individual Case Safety Reports
}

\author{
Alem Zekarias $^{1}$ (D) Sarah Watson ${ }^{1}$ (D) $\cdot$ Sara Hedfors Vidlin $^{1}$ (D) $\cdot$ Birgitta Grundmark $^{1}$ (D)
}

Accepted: 8 September 2020 / Published online: 25 September 2020

(c) The Author(s) 2020

\begin{abstract}
Introduction In late 2019, a new coronavirus-severe acute respiratory syndrome coronavirus 2 (SARS-CoV-2)—was discovered in Wuhan, China, and the World Health Organization later declared coronavirus disease 2019 (COVID-19) a pandemic. Numerous drugs have been repurposed and investigated for therapeutic effectiveness in the disease, including those from "Solidarity," an international clinical trial (azithromycin, chloroquine, hydroxychloroquine, the fixed combination lopinavir/ritonavir, and remdesivir).

Objective Our objective was to evaluate adverse drug reaction (ADR) reporting for drugs when used in the treatment of COVID-19 compared with use for other indications, specifically focussing on sex differences.

Method We extracted reports on COVID-19-specific treatments from the global ADR database, VigiBase, using an algorithm developed to identify reports that listed COVID-19 as the indication. The Solidarity trial drugs were included, as were any drugs reported $\geq 100$ times. We performed a descriptive comparison of reports for the same drugs used in non-COVID-19 indications. The data lock point date was 7 June 2020.

Results In total, 2573 reports were identified for drugs used in the treatment of COVID-19. In order of frequency, the most reported ADRs were electrocardiogram QT-prolonged, diarrhoea, nausea, hepatitis, and vomiting in males and diarrhoea, electrocardiogram QT-prolonged, nausea, vomiting, and upper abdominal pain in females. Other hepatic and kidney-related events were included in the top ten ADRs in males, whereas no hepatic or renal terms were reported for females. COVID19-related reporting patterns differed from non-pandemic reporting for these drugs.
\end{abstract}

Conclusion Review of a global database of suspected ADR reports revealed sex differences in the reporting patterns for drugs used in the treatment of COVID-19. Patterns of ADR sex differences need further elucidation.

\section{Introduction}

In December 2019, a novel $\beta$-coronavirus, severe acute respiratory syndrome coronavirus 2 (SARS-CoV-2), was discovered in Wuhan, China, and soon spread worldwide [1]. By 11 March 2020, the World Health Organization (WHO) declared coronavirus disease 2019 (COVID-19) a pandemic and a public health emergency of international concern [2]. As of 16 June, $>8$ million people were confirmed infected and $>400,000$ had died globally [3]. As

Electronic supplementary material The online version of this article (https://doi.org/10.1007/s40264-020-01000-8) contains supplementary material, which is available to authorized users.

Alem Zekarias

alem.zekarias@who-umc.org

1 Uppsala Monitoring Centre, Uppsala, Sweden

\section{Key Points}

Sex differences in the reporting of adverse drug reactions (ADRs) for COVID-19-related drugs were observed in a global ADR database.

More reports were received for males, which is consistent with their greater risk for COVID-19.

The top reported ADRs for the same drugs also differ by sex.

there were initially no authorized treatments for COVID19 , any drug used in the treatment of the disease could be considered off-label use. Numerous drugs are being repurposed and investigated for therapeutic efficacy. A notable effort launched by the WHO is the international 
"Solidarity" trial, which is evaluating the efficacy of azithromycin, chloroquine, hydroxychloroquine, the fixed-combination lopinavir/ritonavir, and remdesivir in COVID-19 compared with standard of care. As of 3 June 2020, more than 3500 patients from 400 hospitals in 35 countries had been recruited into the trial [4]. The initial protocol included both hydroxychloroquine and chloroquine; however, the WHO announced on 25 May 2020 that the trial had only pursued hydroxychloroquine and on 6 July 2020 that the hydroxychloroquine and lopinavir/ ritonavir arms were being discontinued [4].

The impact of patient sex on COVID-19 outcomes has previously been described [5-7]. An early study investigated the influence of patient sex on morbidity and mortality in both COVID-19 and the earlier severe acute respiratory syndrome (SARS-CoV-1) epidemic in 2002 [6, 8]. The authors concluded that men and women had a similar incidence of the diseases, but that men with COVID-19 or SARS-CoV-1 infection were at higher risk of worse outcomes and death, independent of age [6]. A review of 48 articles representing multiple countries and databases confirmed this finding [7], reporting that males accounted for 55-62\% of hospitalized patients. The sex imbalance was even more pronounced for admissions to intensive care units (ICUs), with a male proportion of $65-74 \%$. Throughout Europe, $73 \%$ of admissions to ICUs were men. Deaths were more common among men than among women in all countries except India and Pakistan [7].

During the initial part of the pandemic, the $>130$ countries within the WHO Programme for International Drug Monitoring (PIDM) began reporting side effects from drugs used to treat COVID-19 into VigiBase, the WHO global database for individual case safety reports. The database contains nearly 23 million reports of suspected adverse drug reactions (ADRs), including reports via the US FDA and EU-wide ADR collecting systems. Methods to monitor and communicate reviews of the COVID19 reporting were rapidly developed within the Uppsala Monitoring Centre, which harbours the database.

When studying sex-related differences related to drug treatment in COVID-19, spontaneous reports of suspected ADRs may complement other data sources, and our study used data retrieved for general monitoring. The vast majority of the reports contain data on age and sex. In VigiBase, reporting rates are higher for women than for men, whereas men have a higher proportion of serious and fatal reports [9].

\section{Aim}

Our aim was to evaluate ADR reporting for drugs used in the treatment of COVID-19 compared with when the same drugs were used in illnesses other than COVID-19, focussing specifically on sex differences.

\section{Methods}

Reports on COVID-19-specific treatments were extracted using an algorithm that had been iteratively developed and manually validated by in-house pharmacovigilance professionals during the initial pandemic phase. The algorithm was primarily created to periodically monitor global reporting patterns of ADRs associated with COVID-19 treatments in general and to share this data with the WHO network. The algorithm scans the indication, free text fields, and laboratory test results of reports in VigiBase up until 7 June 2020 (see Fig. 1 for full flow chart). Drugs from the Solidarity trial were included, as well as the originally announced chloroquine arm and any other drug reported at least 100 times as captured by the algorithm; two drugs, tocilizumab and oseltamivir, fulfilled the latter criteria. We rejected all reports where drugs had been taken as prophylaxis or for which the narrative described negative polymerase chain reaction testing. The extracted data were divided into subsets based on sex: male, female, and unknown (the latter grouping included 'not known', 'not applicable', and missing values). All reports (serious and non-serious as per International Conference on Harmonisation of Technical Requirements for Registration of Pharmaceuticals for Human Use guidance) in which the drugs were reported as 'suspected' or 'interacting' were included [10]. For the background comparison, the same seven drugs when used outside the context of COVID-19, until 7 June 2020, were included. For remdesivir, only-COVID-19 reports were identified because it was not licensed for use before the pandemic occurred. One remdesivir report that lacked sufficient data to classify it as used for COVID-19 or not was included in the background. We used Medical Dictionary for Regulatory Activities $\left(\right.$ MedDRA ${ }^{\circledR}$ ) preferred terms (PTs) for the analysis of reported ADRs [11]. Data for this study were analysed using descriptive statistics. Individual case assessments were not performed for this study.

\section{Results}

A total of 2573 reports were identified with an indication of COVID-19; $93 \%$ of these reports included one or more of the drugs hydroxychloroquine, chloroquine, lopinavir/ritonavir, azithromycin, or remdesivir. In total, $39 \%$ were for women (1003 reports), 55\% were for men (1426 reports), and 6\% (144) did not report the biological sex. The reports originated from Europe (63\%), Eastern Mediterranean (16\%), the Americas (12\%), Western Pacific (6\%), South-East Asia $(2 \%)$, and Africa $(<1 \%)$. In total, 58 and $44 \%$ of reports for males and females, respectively, were classified as serious. Hydroxychloroquine, azithromycin, lopinavir/ritonavir, remdesivir, and tocilizumab were all reported more often in 


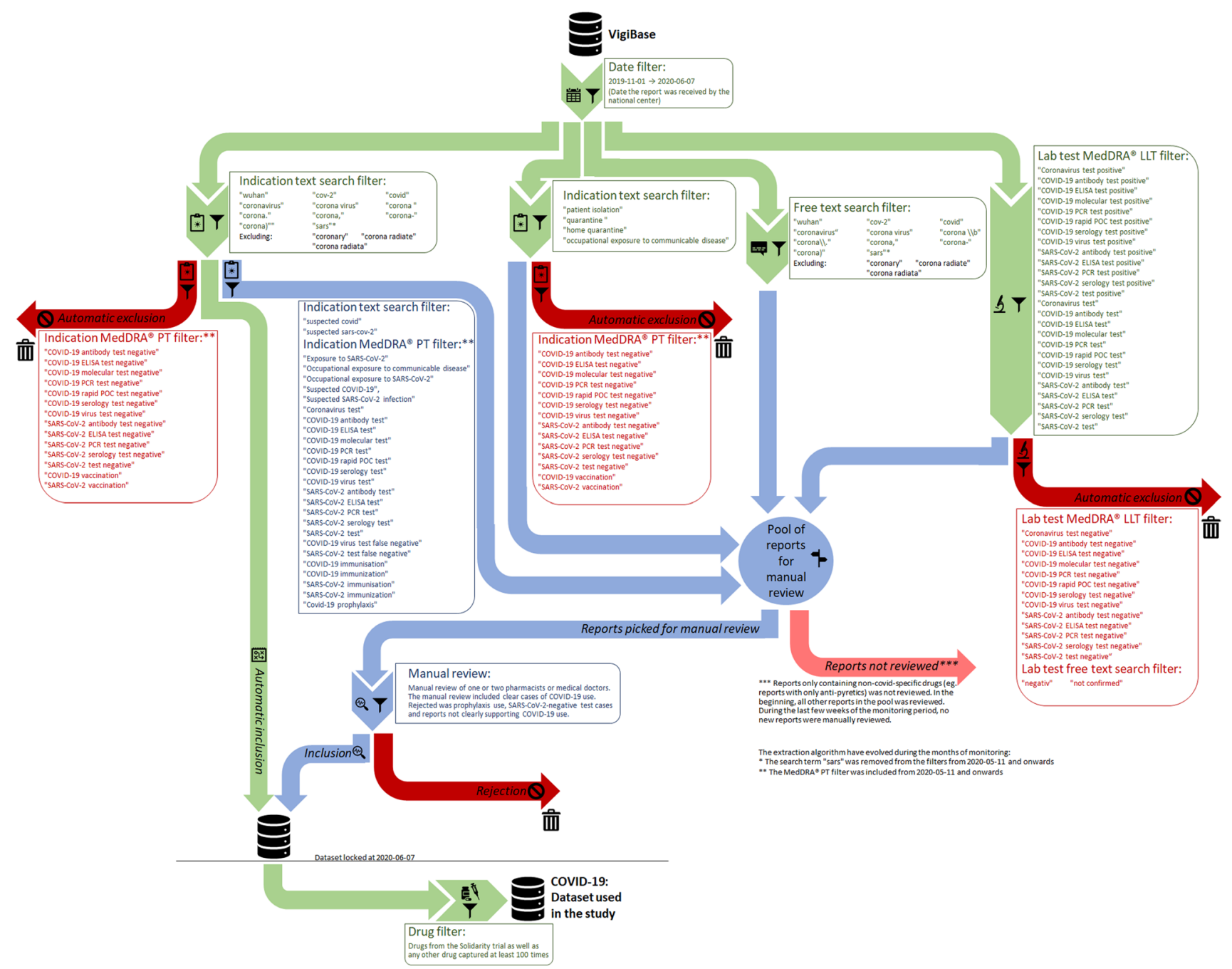

Fig. 1 Flow chart of the scanning algorithm used to create the dataset for the study. All parts above the line were developed iteratively approximately every or every other week, from April 2020, with every iteration adding a batch of reports to the dataset. The filters were adjusted along the way according to (1) needs seen when manually reviewing reports, (2) needs emerging from a growing dataset,

men in the COVID-19 subset $(P=0.0005$ for azithromycin, $P<0.0001$ for the rest, against the null hypothesis that both sexes were reported at the same rate). Only lopinavir/ritonavir exhibited this male predominance otherwise in VigiBase $(P<0.0001$ for all, remdesivir excluded because no cases existed). In contrast, for chloroquine, the proportion of reports was $54 \%$ (190 reports) for females versus $42 \%$ (147 reports) for males $(P=0.0012)$, similar to the reporting pattern in the rest of VigiBase (58 vs. 39\%; $P<0.0001$ ). Reports relating to oseltamivir were almost equally distributed between the sexes (51\% $[n=66]$ male vs. $49 \%[n=63]$ female), whereas the female proportion was higher ( $52 \mathrm{vs}$. $40 \% ; P<0.0001)$ in the rest of VigiBase. See Fig. 2 for a comparison of the datasets. The most commonly reported
(3) new MedDRA ${ }^{\circledR}$ versions released. The figure represents the algorithm at the point of data lockdown, with major adjustments marked. ELISA enzyme-linked immunosorbent assay, LLT Low Level Term, MedDRA Medical Dictionary for Regulatory Activities, PCR polymerase chain reaction, $P O C$ Point of Care, $P T$ preferred term

drugs were hydroxychloroquine (864 reports), azithromycin (478), and lopinavir/ritonavir (309) in males and hydroxychloroquine (612), azithromycin (404), and lopinavir/ritonavir (160) in females.

Chloroquine was the only drug for which more reports concerned females, with 28 and $42 \%$ of the reports classified as serious for females and males, respectively. In those aged $65-74$ years, $19 \%$ of chloroquine reports concerned males and $11 \%$ concerned females. One country contributed $71 \%$ of the female reports and $45 \%$ of the male reports. Azithromycin was co-reported as suspected or interacting in 74 and $51 \%$ of the female and male chloroquine reports, respectively. 


\section{Adverse Drug Reaction Profiles in COVID-19 and Non-COVID-19 Use}

The most commonly reported ADRs with treatments for COVID-19, in order of frequency, were electrocardiogram QT-prolonged, diarrhoea, nausea, hepatitis, and vomiting in males and diarrhoea, electrocardiogram QT-prolonged, nausea, vomiting, and upper abdominal pain in females (Fig. 3). The top ADR reporting spectrum for drugs used for COVID-19 differed from that for the same drugs used for other indications, with only gastrointestinal reactions remaining the same. The ten most reported terms in COVID19-related use in males but not females were hepatitis, other hepatic enzyme aberrations, and renal injury (Table 1 in the electronic supplementary material [ESM]). These were mostly related to the use of hydroxychloroquine and, to a lesser extent, the use of lopinavir/ritonavir and remdesivir. Conversely, psycho-neurological terms such as insomnia, headache, vertigo, and blurred vision were in the top ten reports for females. No cardiac, hepatic, or renal terms were included in the ten most reported ADRs when the same drugs were used for other indications.

Sex distribution differed most between COVID-19-related and other indications in reports regarding the use of chloroquine. The most commonly reported ADRs were similar for women and men except for the term electrocardiogram QT-prolonged, which was reported in 31\% of male reports and only $19 \%$ of female reports $(P=0.013)$.

\section{Discussion}

The male predominance in COVID-19 ADR reporting is consistent with observations of a greater risk of COVID19 in males than in females. However, the male predominance in reporting deviates from the overall reporting into VigiBase. For chloroquine and oseltamivir, in which male COVID-19-related reporting was less dominant, the finding appeared to be driven mainly by reporting from two countries. Interestingly, the reaction electrocardiogram QT-prolonged was more commonly reported for men than for women, despite this event being known to be twice as common in women than in men $[12,13]$. The observation that acute hepatitis, different hepatic enzyme aberrations, and renal injury were among the most common events in male COVID-19 reporting than in both female COVID-19 reporting and non-COVID-19 reporting may be related to the symptoms and severity of the underlying disease and the sex differences in treatment reactions. The global public attention and the potential for cardiac side effects with hydroxychloroquine and chloroquine may have influenced this reporting pattern for electrocardiogram QT-prolonged [14].The extent of rational or irrational differences in COVID-19-related drug use between the sexes and subsequent suspected ADR patterns cannot yet be fully determined from existing data. It is possible that factors other than sex influence ADR patterns, given the other known risk factors (such as age and obesity) for COVID-19 that

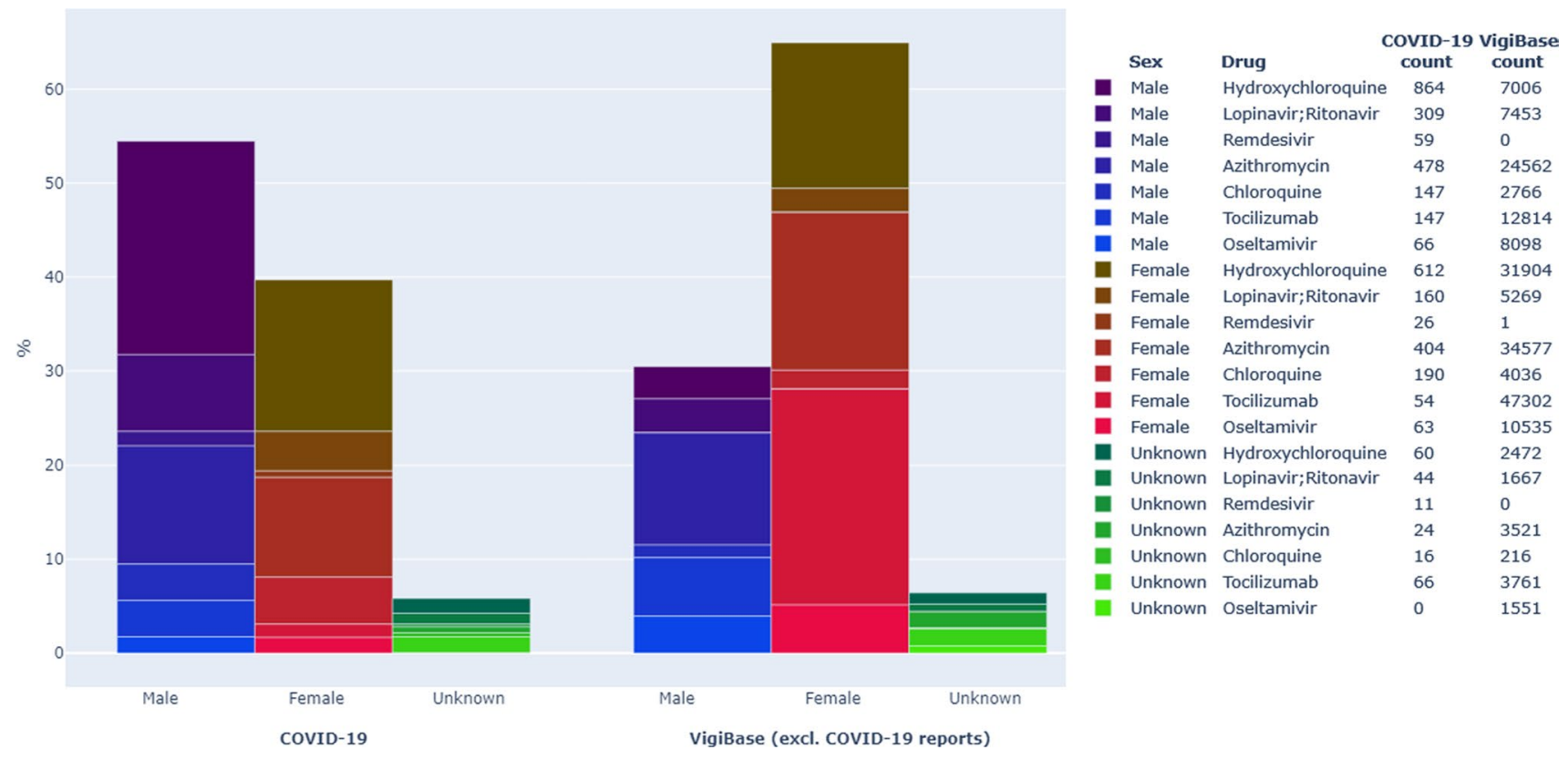

Fig. 2 Proportions of VigiBase reporting by sex for drugs included in the study. Bars to the left show the COVID-19 subset of VigiBase; bars to the right show the same drugs used for other indications. The legend shows the number of reports for each drug and subset 


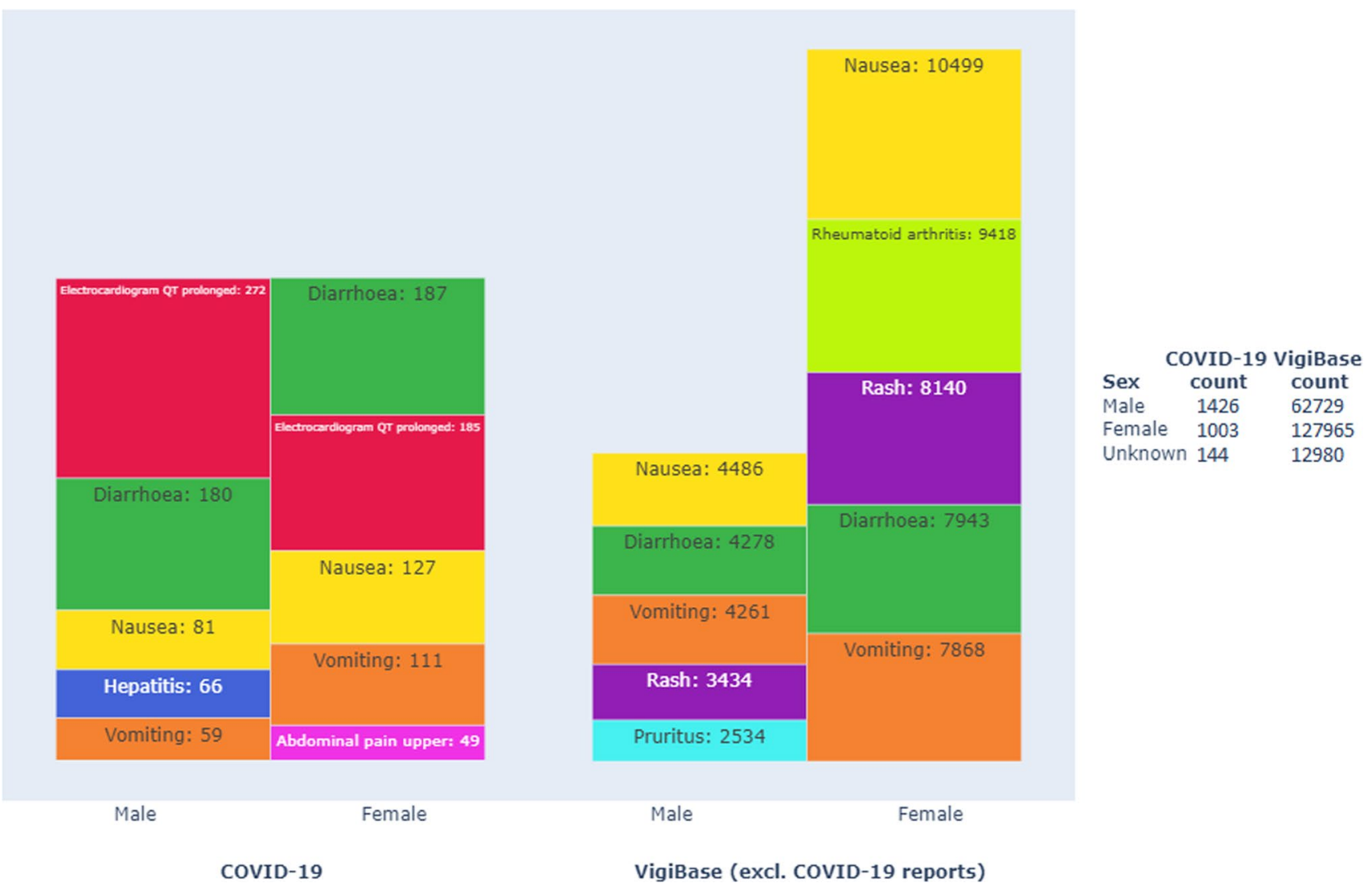

Fig. 3 Top five reported adverse drug reactions (MedDRA ${ }^{\circledR}$ preferred terms), with number of reported instances, for the included drugs used in treating COVID-19 (left) vs. for the same drugs used in other indications (right), separated by sex. Reports with unknown sex and the MedDRA ${ }^{\circledR}$ preferred terms off-label use, intentional product use

can also influence the occurrence of ADRs. Other contributing factors could potentially be found in the immense information flow, e.g. on theoretically suitable treatments, both from scientific sources and from the lay media, present since the pandemic began. This study shows that ADR databases such as VigiBase provide rich opportunities for research on aspects of reported side effects beyond the classic causality investigations for which the data for this study were originally gathered. Limitations of VigiBase data, as for all spontaneous data, include underreporting, variability in the nature and completeness of reports between different countries of origin, lack of denominator data of drug use, and the fact that the reports only describe suspicions of causality rather than proven associations. We acknowledge the risk of misclassification bias given the possibility of incomplete reporting on indication; however, COVID-19 reports can potentially be identified by other means, such as co-reported medications. issue, and drug ineffective are excluded. The size of the box represents the proportions within the COVID-19 and VigiBase datasets, respectively. Reports may contain more than one reported preferred term. The legend shows the number of reports for each subset

\section{Conclusion}

Our review of a global spontaneous database of suspected ADR reports revealed sex differences in the reporting patterns for drugs used in the treatment of COVID-19. The increased risk for males of severe COVID-19 disease is reflected in a male predominance of ADR reports in drugs used in the COVID-19 indication. Notably, some hepatic and renal reactions and QT prolongation were more commonly observed in male reports. Further explorations of sex differences in reporting patterns and additional in-depth analyses are warranted as reporting continues during the pandemic.

Acknowledgements The authors are indebted to the national centres that make up the WHO PIDM and provide reports to VigiBase. However, the opinions and conclusions of this study are not necessarily those of the various centres nor of the WHO.

\section{Declarations}

Funding No sources of funding were used to conduct this study or prepare this manuscript. 
Conflicts of Interest Alem Zekarias, Sarah Watson, Sara Hedfors Vidlin, and Birgitta Grundmark have no conflicts of interest that are directly relevant to the content of this study.

Ethics Approval Not applicable.

Consent to Participate Not applicable.

Consent for Publication Not applicable.

Availability of Data and Materials The datasets generated and analysed during the current study are not publicly available because of agreements between contributors of data to the database used (VigiBase) and the custodian of this database. National centres (mainly national drug regulatory authorities) constituting the WHO PIDM contribute data to VigiBase, and the Uppsala Monitoring Centre is the custodian in its capacity as the WHO collaborating centre for international drug monitoring. Some subsets of the data may be available from the corresponding author on reasonable request.

Code Availability Code is unavailable.

Author Contributions All authors contributed to this manuscript.

Open Access This article is licensed under a Creative Commons Attribution-NonCommercial 4.0 International License, which permits any non-commercial use, sharing, adaptation, distribution and reproduction in any medium or format, as long as you give appropriate credit to the original author(s) and the source, provide a link to the Creative Commons licence, and indicate if changes were made. The images or other third party material in this article are included in the article's Creative Commons licence, unless indicated otherwise in a credit line to the material. If material is not included in the article's Creative Commons licence and your intended use is not permitted by statutory regulation or exceeds the permitted use, you will need to obtain permission directly from the copyright holder. To view a copy of this licence, visit http://creativecommons.org/licenses/by-nc/4.0/.

\section{References}

1. Guo Y-R, Cao Q-D, Hong Z-S, Tan Y-Y, Chen S-D, Jin H-J, et al. The origin, transmission and clinical therapies on coronavirus disease 2019 (COVID-19) outbreak-an update on the status. Mil Med Res. 2020;7:11.
2. WHO. Coronavirus disease (COVID-2019) situation reports. 2020. January 31 [Internet]. https://www.who.int/docs/defaultsource/coronaviruse/situation-reports/20200131-sitrep-11-ncov. pdf?sfvrsn=de7c0f7_4. Accessed 11 June 2020.

3. COVID-19 dashboard by the Center for Systems Science and Engineering (CSSE) at Johns Hopkins University (JHU) [Internet]. https://www.arcgis.com/apps/opsdashboard/index.html\#/ bda7594740fd40299423467b48e9ecf6. Accessed 12 June 2020.

4. "Solidarity" clinical trial for COVID-19 treatments [Internet]. https://www.who.int/emergencies/diseases/novel-coronaviru s-2019/global-research-on-novel-coronavirus-2019-ncov/solid arity-clinical-trial-for-covid-19-treatments. Accessed 10 Aug 2020.

5. Gebhard C, Regitz-Zagrosek V, Neuhauser HK, Morgan R, Klein SL. Impact of sex and gender on COVID-19 outcomes in Europe. Biol Sex Differ. 2020;11:29.

6. Jin J-M, Bai P, He W, Wu F, Liu X-F, Han D-M, et al. Gender differences in patients with COVID-19: focus on severity and mortality. Front Public Health. 2020;8:152.

7. Rozenberg S, Vandromme J, Charlotte M. Are we equal in adversity? Does Covid-19 affect women and men differently? Maturitas. 2020;138:62-8.

8. Drosten C, Günther S, Preiser W, van der Werf S, Brodt $\mathrm{H}-\mathrm{R}$, Becker $\mathrm{S}$, et al. Identification of a novel coronavirus in patients with severe acute respiratory syndrome. N Engl J Med. 2003;348:1967-76.

9. Watson S, Caster O, Rochon PA, den Ruijter H. Reported adverse drug reactions in women and men: aggregated evidence from globally collected individual case reports during half a century. EClinicalMedicine. 2019;17:100188.

10. ICH harmonised tripartite guideline post-approval safety data management: definitions and standards for expedited reporting E2D [Internet]. https://database.ich.org/sites/default/files/E2D_ Guideline.pdf. Accessed 10 Aug 2020.

11. Medical Dictionary for Regulatory Activities [Internet]. https:// www.meddra.org/sites/default/files/guidance/file/000026_meddr a_best_practices_2018_0.pdf. Accessed 22 June 2020.

12. Miranda D, McMain C, Smith A. Medication-induced QTinterval prolongation and Torsades de Pointes. US Pharm. 2011;36(2):HS-2HS-8.

13. Wolbrette D, Patel H. Arrhythmias and women. Current Opinion in Cardiology. 1999;14(Issue 1):36.

14. Public warning on the use of chloroquine's [Internet]. https:// www.fda.gov/drugs/drug-safety-and-availability/fda-cautionsagainst-use-hydroxychloroquine-or-chloroquine-covid-19-outsi de-hospital-setting-or. Accessed 22 June 2020. 